\title{
BMJ Open Safety and efficacy of stereotactic body radiation therapy combined with $S-1$ simultaneously followed by sequential S-1 as an initial treatment for locally advanced pancreatic cancer (SILAPANC) trial: study design and rationale of a phase II clinical trial
}

Xiaofei Zhu, Xiaoping Ju, Fei Cao, Fang Fang, Shuiwang Qing, Yuxin Shen, Zhen Jia, Yangsen Cao, Huojun Zhang

To cite: Zhu X, Ju X, Cao F, et al. Safety and efficacy of stereotactic body radiation therapy combined with S-1 simultaneously followed by sequential S-1 as an initial treatment for locally advanced pancreatic cancer (SILAPANC) trial: study design and rationale of a phase II clinical trial. BMJ Open 2016;6: 013220. doi:10.1136/bmjopen-2016013220

- Prepublication history for this paper is available online. To view these files please visit the journal online (http://dx.doi.org/10.1136/ bmjopen-2016-013220).

$\mathrm{XZ}$ and $\mathrm{XJ}$ contributed equally to this article.

Received 27 June 2016 Revised 23 September 2016 Accepted 8 November 2016

CrossMark

For numbered affiliations see end of article.

Correspondence to Dr Huojun Zhang; chyyzhj@163.com

\section{ABSTRACT}

Introduction: Upfront surgeries are not beneficial to most patients with pancreatic cancer. Therefore, more emphasis has been placed chemoradiotherapy in locally advanced pancreatic cancer recently. Gemcitabine-based regimens or FOLFIRINOX (a chemotherapy regimen including leucovorin, 5-FU, irinotecan, oxaliplatin) has been proven as a standard chemotherapy in pancreatic cancer. However, severe toxicities may prevent the completion of chemotherapy. S-1 has showed better objective response rates, similar overall survival rates and progression-free survival rates compared with gemcitabine, revealing that $\mathrm{S}-1$ may be a potential candidate in treating pancreatic cancer, especially for patients refractory to gemcitabine. Additionally, stereotactic body radiation therapy with Cyberknife could provide better efficacy than conventional radiotherapy in pancreatic cancer. Therefore, Cyberknife with S-1 simultaneously followed by sequential S-1 as an initial treatment may bring about favourable outcomes but needs further studies.

Methods and analysis: The S-1 as an initial treatment for locally advanced pancreatic cancer (SILAPANC) trial is a prospective, single-centre, one armed ongoing study. 190 eligible patients are required to initially receive Cyberknife with 1 cycle of S-1 simultaneously. After the concurrent chemoradiotherapy, 2 or 3 cycles of S-1 are sequentially given. Doses and fractions depend on the locations and volumes of tumours and the adjacent organs at risk. S-1 is taken orally, 2 times a day, at a dose of $80 \mathrm{mg} / \mathrm{m}^{2}$ for 28 days, followed by a 14-day interval. The primary objectives are overall survival and 1-year, 2-year, 3-year, 4-year and 5-year overall survival rates. The secondary objectives are cancerspecific survival, progression-free survival, time to progression, local control rates, clinical benefit rates, radiation-induced acute and late toxicities, adverse

\section{Strengths and limitations of this study}

- This is the first study to evaluate the feasibility and efficacy of stereotactic body radiation therapy with Cyberknife combined with S-1 followed by sequential S-1 initially treated in patients with locally advanced pancreatic cancer.

- Owing to no optimal treatment in locally advanced pancreatic cancer and high adverse effects of the standard chemotherapy, alternative combination therapies with favourable efficacy and low toxicities are urgent. Cyberknife and S-1 have been proved effective in pancreatic cancer with low incidences of adverse effects.

- This is a single-centre, one-arm study. Comparison with other treatment needs to be further investigated after the phase II trial.

- Loss of participants at follow-up is inevitable. Hence, recruitment and duration of the study may be extended for availability of data for analysis.

effects of chemotherapy and quality of life of patients. Besides, variables most predictive of prognosis would be identified via multivariate methods.

Ethics and dissemination: Approvals have been granted by the Changhai Hospital Ethics Committee (CHEC-2016-032-01). The results will be disseminated in peer-reviewed journals and at conferences.

Trial registration number: NCT02704143;

Pre-results.

\section{INTRODUCTION}

Although the incidence rate of pancreatic cancer was not as high as that of other 
gastrointestinal cancer in China, cancer mortalities in men and women ranked the sixth and seventh, respectively, in 2013, with surprisingly low 5-year survival rates $(<5 \%){ }^{1}$ Among patients first diagnosed with pancreatic cancer, only $15-20 \%$ of these patients were suitable for surgery, ${ }^{2}$ and the 5-year survival rate of patients with R0 resection remained $<20 \%{ }^{3-5}$

Therefore, better efficacy could not be obtained via surgeries alone, resulting in great emphasis on adjuvant chemoradiotherapy. In 1997, gemcitabine had been confirmed to be the standard chemotherapy for pancreatic cancer. ${ }^{6}$ However, it has not been proven whether gemcitabine can significantly improve prognosis in long-term follow-ups, especially when some patients were refractory to gemcitabine. Hence, there was an urgent need for the development of more effective chemotherapy.

$\mathrm{S}-1$ is the prodrug of 5-fluorouracil (5-FU), which comprises of tegafur, gimeracil (dihydropyrimidine dehydrogenase inhibitor) and oteracil (inhibitor of phosphorylation in the gastrointestinal tract), with a ratio of 1:0.4:1. The first phase II clinical trials revealed good clinical efficacy with S-1. ${ }^{7}$ In GEST, S-1 had better objective response rates than gemcitabine. In addition, S-1 was not inferior to gemcitabine in terms of overall survival (OS) rates and progression-free survival (PFS) rates. Furthermore, significant improvement of PFS rates could be achieved by the combination of S-1 and gemcitabine. ${ }^{8}$ There was no difference between the incidence rates of the adverse effects of S-1 and gemcitabine. Therefore, S- 1 was an alternative for treating locally advanced or metastatic pancreatic cancer, especially for those resistant to gemcitabine. Although there are no phase III studies on S-1, phase II studies have already shown better disease control rates (52-58\%), median OS time (4.5-6.3 months) and tolerable adverse effects in gemcitabine-resistant advanced pancreatic cancers treated with $\mathrm{S}-1 .{ }^{9-10}$

However, few encouraging results were gained with the combination of S-1 and the other medications. ${ }^{811-13}$ As a result S-1 combined with radiotherapy has been gradually applied for the treatment of pancreatic cancer. Besides, 5-FU has been proven to be radiosensitive; thus, improving clinical efficacy. ${ }^{14}$

Compared with conventional radiation, a singlefraction dose and the total dose of the target volume can be increased in stereotactic body radiation therapy (SBRT). In addition, doses of organs at risk would be reduced; thus, effectively improving local control rates (LCR) and reducing radiation-related toxicities. ${ }^{15}{ }^{16}$ Shorter courses of SBRT also enhance patient's compliance and render the initial other treatments on schedule possible. ${ }^{16}$ Previous studies on SBRT combined with other chemotherapy regimens are presented in table 1 . Nevertheless, few studies have focused on S-1 combined with SBRT for locally advanced pancreatic cancer. Hence, the feasibility of combining S-1 and SBRT needs to be further confirmed. Based on our experience in treating locally advanced pancreatic cancer, SBRT combined with S-1 followed by sequential S-1 as the initial treatment for locally advanced pancreatic cancer has been proposed to evaluate its clinical efficacy.

\section{METHODS}

\section{Study design}

This is a single-centre, prospective, single-arm and phase II clinical trial designed and sponsored by the Department of Radiation Oncology of Changhai Hospital, which evaluated the safety and efficacy of combining Cyberknife with $\mathrm{S}-1$ followed by sequential $\mathrm{S}-1$ as an initial treatment in patients with locally advanced pancreatic cancer. After obtaining the patients' written informed consents, their information about baseline characteristics, individual treatment plans and follow-ups would be processed by database administrators responsible for this clinical trial. Although FOLFIRINOX and gemcitabine-based chemotherapy have been proven as a standard chemotherapy, side effects, especially gastrointestinal and haematological toxicities, may hamper the completion of full treatment with these drugs, and probably even result in reduced quality of life. Therefore, the advantage of S-1 in this case due to its features may display a favourable tolerability and safety profile. Presumably, this yields an innovative therapy, if deemed favourable, compared with conventional chemotherapy; and this could be another alternative, or even a recommended therapy, when patients are vulnerable or resistant to gemcitabine. The main rationale for the active recruitment of the $\mathrm{S}-1$ as an initial treatment for locally advanced pancreatic cancer (SILAPANC) trial is to demonstrate the good tolerability of treatment with SBRT via Cyberknife combined with S-1, followed by sequential S-1 and to assess a potential therapeutic benefit based on the prognosis of patients.

\section{Study objectives}

The SILAPANC trial aims to investigate the efficacy and adverse effects of Cyberknife with S-1 in patients with locally advanced pancreatic cancer. The primary objectives are:

1. To evaluate the prognosis of patients with locally advanced pancreatic cancer after radiochemotherapy;

2. To determine adverse effects attributable to Cyberknife or S-1.

The secondary objectives are:

1. To analyse the quality of life of patients with locally advanced pancreatic cancer treated with Cyberknife combined with $\mathrm{S}-1$;

2. To demonstrate the potential factors associated with the safety and prognosis of patients with locally advanced pancreatic cancer after radiochemotherapy.

\section{Participants and eligibility}

To be eligible for inclusion in the SILAPANC trial, all patients with clinical suspicion for pancreatic cancer, as presented in the imaging studies, were required to undergo pathological examinations. If deemed necessary, further high-quality dedicated imaging of the 
Table 1 Recent studies evaluating SBRT in pancreatic cancer

\begin{tabular}{|c|c|c|c|c|c|c|}
\hline Study & Patients & Dose & $\begin{array}{l}\text { Median OS } \\
\text { (months) }\end{array}$ & 1 year OS rate & Toxicity & Chemotherapy \\
\hline Chuong et al ${ }^{17}$ (2013) & $73 \mathrm{BR}$ or LA & $5-10 \mathrm{~Gy} \times 5 f$ & 16.4 BR; 15 LA & $\begin{array}{l}72.2 \% \text { BR; } \\
68.1 \% \text { LA }\end{array}$ & $\begin{array}{l}5 \% \text { grade } 3 \\
\text { (late) }\end{array}$ & 3 cycles GTX \\
\hline Herman et al ${ }^{18}$ (2014) & 49 LA & $6.6 \mathrm{~Gy} \times 5 f$ & 13.9 & $59 \%$ & $\begin{array}{l}2 \% \text { acute grade } \\
\geq 2 \\
11 \% \text { late grade } \\
\geq 2\end{array}$ & GEM followed by SBRT \\
\hline Mahadevan et al ${ }^{19}$ (2010) & 36 LA & $8-12$ Gy×3f & 20 & - & $\begin{array}{l}33 \% \text { grade } 1-2 \\
8 \% \text { grade } 3\end{array}$ & Post-SBRT GEM \\
\hline Koong et $a^{20}$ (2004) & 15 LA & $15-25$ Gy×1f & 11 & - & $\begin{array}{l}33 \% \text { grade } 1-2 \\
0 \% \text { grade } 3\end{array}$ & None \\
\hline Koong et $a^{\Gamma}$ (2005) & 16 LA & 25 Gy×1f (boost) & 8.3 & $15 \%$ & $\begin{array}{l}69 \% \text { grade } 1-2 \\
12.5 \% \text { grade } 3\end{array}$ & 5-FU with EBRT prior to boost \\
\hline Hoyer et $a^{P^{1}}$ (2005) & 22 LA & $15 \mathrm{~Gy} \times 3 f$ & 5.4 & $5 \%$ & $\begin{array}{l}79 \% \text { grade } 2 \\
4.5 \% \text { grade } 4\end{array}$ & None \\
\hline Schellenberg et $a^{P^{2}}$ (2008) & 16 LA & $25 \mathrm{~Gy} \times 1 f$ & 11.4 & $50 \%$ & $\begin{array}{l}19 \% \text { acute } \\
\text { toxicity } \\
47 \% \text { late toxicity }\end{array}$ & $\begin{array}{l}1 \text { cycle of induction GEM+post-SBRT } \\
\text { GEM }\end{array}$ \\
\hline Schellenberg et $a^{23}$ (2011) & 20 LA & $25 \mathrm{~Gy} \times 1 f$ & 11.8 & $50 \%$ & $\begin{array}{l}15 \% \text { grades } 1-2 \\
5 \% \geq \text { grade } 3\end{array}$ & $\begin{array}{l}1 \text { cycle of induction GEM+post-SBRT } \\
\text { GEM }\end{array}$ \\
\hline Mahadevan et a ${ }^{4}$ (2011) & 39 LA & 8-12 Gy×3f & 20 & - & $\begin{array}{l}41 \% \text { grade } 1-2 \\
0 \% \text { acute grade } \\
39 \% \text { late grade } \\
3\end{array}$ & 2 cycles induction GEM \\
\hline Polistina et $a P^{5}$ (2010) & 23 LA & $10 \mathrm{~Gy} \times 3 f$ & 10.6 & $39.1 \%$ & $\begin{array}{l}20 \% \text { grade } 1 \\
0 \% \text { grade } 2\end{array}$ & 6 weeks of induction GEM \\
\hline Rwigema et $a^{P^{6}}$ (2011) & $\begin{array}{l}71 \text { (LA, LR, RPM, } \\
\text { MD) }\end{array}$ & $24 \mathrm{~Gy} \times 1 f$ & 10.3 & $41 \%$ & $\begin{array}{l}39.5 \% \text { grade } 1- \\
2 \\
4.2 \% \text { grade } 3\end{array}$ & $\begin{array}{l}90 \% \text { received chemotherapy (various } \\
\text { regimens) }\end{array}$ \\
\hline Gurka et $a R^{7}$ (2013) & $10 \mathrm{LA}$ & 5 Gy $\times 5 f$ & 12.2 & - & $0 \%$ grade 3 & $\begin{array}{l}1 \text { cycle GEM prior to SBRT, } 6 \text { cycles } \\
\text { GEM total }\end{array}$ \\
\hline Didolkar et $a P^{8}$ (2010) & 85 LA or LR & $5-10 \mathrm{~Gy} \times 3 f$ & 18.6 & $50 \%$ & $22.3 \% \geq$ grade 3 & Post-SBRT GEM \\
\hline Goyal et a $f^{9}$ (2012) & 19 LA or LR & $\begin{array}{l}20-25 \mathrm{~Gy} \times 1 f \\
8-10 \mathrm{~Gy} \times 3 f\end{array}$ & 14.4 & $56 \%$ & $\begin{array}{l}11 \% \text { grade } 1-2 \\
16 \% \text { grade } 3\end{array}$ & $68 \% 5-\mathrm{FU}$ or GEM based \\
\hline
\end{tabular}


pancreas should be performed before patients are enrolled into the study and undergo any study-related procedures. Biopsies were performed with fine-needle aspiration via endoscopic ultrasound by experienced gastroenterologists. Specimen sections would be evaluated by two independent pathologists. After confirmed diagnosis of locally advanced pancreatic cancer by pathological examinations, patients should have the willingness and ability to provide an informed consent and comply with subsequent treatment plans, tests and other study procedures.

The following inclusion and exclusion criteria would be employed to preserve high internal validity and reduce risks of SBRT or S-1-induced adverse effects. In our study, locally advanced pancreatic cancer included borderline resectable or unresectable tumours without metastatic pancreatic cancer.

Inclusion criteria:

1. Borderline resectable: ${ }^{30}$

A. Pancreatic head/uncinate process:

I. I. Solid tumour contacts with the common hepatic artery without extension to the coeliac axis or hepatic artery bifurcation, allowing for a safe and complete resection and reconstruction;

II. Solid tumour contact with the superior mesenteric artery of $\leq 180^{\circ}$;

III. The presence of variant arterial anatomy (such as accessory right hepatic artery, replaced right hepatic artery, replaced common hepatic artery, and the origin of replaced or accessory artery), and the presence and degree of tumour contact should be noted, if present, as it may affect surgical planning.

B. Pancreatic body/tail:

I. I. Solid tumour contact with the coeliac axis of $\leq 180^{\circ}$;

II. Solid tumour contact with the coeliac axis of $\geq 180^{\circ}$ without the involvement of the aorta, and with intact and uninvolved gastroduodenal artery.

C. Solid tumour contact with superior mesenteric veins or portal veins of $>180^{\circ}$, contact of $\leq 180^{\circ}$ with contour irregularity of the vein or thrombosis of the vein, but with a suitable vessel proximal and distal to the site of involvement, allowing for the safe and complete resection of the tumour and vein reconstruction;

D. Solid tumour contact with the inferior vena cava.

2. Unresectable without metastasis: ${ }^{30}$

A. Pancreatic head/uncinate process:

I. I. Solid tumour contact with the superior mesenteric artery $>180^{\circ}$;

II. Solid tumour contact with the coeliac axis $>180^{\circ}$;

III. Solid tumour contact with the first jejunal superior mesenteric artery branch;
IV. Unreconstructible superior mesenteric vein/ portal vein due to involvement or occlusion (can be due to tumour or bland thrombus);

V. Contact with most proximal draining jejunal branch into the superior mesenteric vein.

B. Pancreatic body/tail:

I. I. Solid tumour contact of $>180^{\circ}$ with the superior mesenteric artery branch;

II. Solid tumour contact with the coeliac axis and aortic involvement;

III. Unreconstructible superior mesenteric vein/ portal vein to tumour involvement or occlusion (can be due to tumour or bland thrombus).

3. Age ranging from 18 to 75 years old;

4. Karnofsky Performance Score (KPS) $\geq 70$;

5. Normal renal function (serum creatinine $\leq 2.0 \mathrm{mg} / \mathrm{dL}$ );

6. Normal liver function (serum total bilirubin $\leq 3.0 \mathrm{mg} / \mathrm{dL}$, serum aspartate transaminase (AST) $\leq 2.5$ of the upper limit of normal, serum alanine transaminase (ALT) $\leq 2.5$ of the upper limit of normal);

7. Routine blood test: white cell count (WCC) $\geq 3.5 \times 10^{9} / \mathrm{L}$, neutrophils $\geq 1.5 \times 10^{9} / \mathrm{L}$, haemoglobin $\geq 80 \mathrm{~g} / \mathrm{L}$, and platelet $\geq 70 \times 10^{9} / \mathrm{L}$.

Exclusion criteria:

1. Ampulla of Vater cancer;

2. Metastatic pancreatic cancer;

3. Patients who had surgeries, chemotherapy or other treatments prior to SBRT;

4. Patients under the age of 18 or above the age of 75 ;

5. $\mathrm{KPS}<70$;

6. Gastrointestinal inflammation or other diseases (especially active inflammatory bowel disease, nonhealing peptic ulcer, gastrointestinal bleeding or perforation within 6 months);

7. Impaired organ functions:

A. Heart failure (New York Heart Association (NYHA) III-IV);

B. Respiratory failure;

C. Renal insufficiency (serum creatinine $>2.0 \mathrm{mg} / \mathrm{dL}$ );

D. Hepatic insufficiency (serum total bilirubin $>3.0 \mathrm{mg} / \mathrm{dL}$, serum AST $>2.5$ of the upper limit of normal, serum ALT>2.5 of the upper limit of normal or Child-Pugh class B or C);

E. Routine blood test: WCC $<3.5 \times 10^{9} / \mathrm{L}$, neutrophils $<1.5 \times 10^{9} / \mathrm{L}, \quad$ haemoglobin $<80 \mathrm{~g} / \mathrm{L}$, platelet $<70 \times 10^{9} / \mathrm{L}$ or other haematological diseases;

F. Severe nervous system diseases.

8. Pregnant women or lactating women;

9. Patients enrolled in other clinical trials;

10. Patients who did not provide an informed consent.

Owing to better diagnostic yield, safety and the potential lower risk of peritoneal seeding, endoscopic ultrasound-guided fine-needle aspiration is preferred for all patients suspected of pancreatic cancer. However, patients with high risks of bleeding, pancreatitis or pancreatic fistula were not recommended to receive 
biopsies. Therefore, it is crucial and mandatory to establish the clinical diagnosis of pancreatic cancer with cautiousness by the multidisciplinary team based on medical histories and all kinds of tests before the following treatment.

\section{Pretreatment assessment}

Potential participants with locally advanced pancreatic cancer confirmed by initial screening were required to undergo a detailed pretreatment assessment, in order to exclude any conditions contradictory to SBRT and S-1. Hence, participants would receive personal interviews with physicians for a detailed explanation of the whole study and related treatments. In addition, written informed consents must be provided prior to the patients' participation in the study, stating their willingness to be treated according to the study protocol. Furthermore, it is important for these patients to complete the required laboratory tests and other examinations for the evaluation of their medical conditions, including blood routine tests, liver and renal function tests, coagulation function tests, tumour markers, physical examinations and KPS scores.

After collection of data regarding pretreatment assessment, this information would be carefully checked and sent to the designers for the final approval of the study enrolment and verification of the diagnosis. After successful enrolment into the study, the baseline quality of life of the participants will be evaluated before treatment via questionnaires (European Organisation for Research and Treatment of Cancer Quality of Life Questionnaires, EORTC QLQ-PAN26 and QLQ-C30).

\section{Withdrawal of participants}

Participants could withdraw from the study any time for any reasons without any consequences. In addition, investigators are required to follow-up the whole treatment in case of radiochemotherapy-related severe adverse effects, in which investigators would stop the treatment temporarily or even exclude patients from the study. Patients who are intolerable to the treatment would definitely receive other alternative therapies based on the guidelines and experience of the multidisciplinary team. For every participant who withdraws from the study, the reasons for withdrawal from treatment should be recorded in details in the database.

\section{Ethical approval}

This study complies with the current Declaration of Helsinki, and the principles of Good Clinical Practice guidelines. This clinical trial has been registered and entered in the clinicaltrials.gov database (NCT02704143). This trial will also be carried out in keeping with local legal and regulatory requirements.

Prior to enrolment, the potential candidates would receive information on the study both verbally and in writing. They would be given 1-week to decide whether to participate into the study. Thereafter, informed consents, during which a physician will explain the nature, scope and possible consequences of the trial to the patient, is obtained from each patient. The investigators will not assume any demands, including publishing or reporting of individual patient's data, especially data required for this clinical trial, until a valid consent has been obtained. Patients' data would be kept strictly confidential within the study, but their pseudonymous medical records and information would be extracted from the database and reviewed for trial purposes by authorised individuals other than their treating physicians.

\section{Study procedures}

Trial overview

After the successful assessment, the participants would be assigned into the treatment group. Individualised treatment plans would be made after the simulation, and these would be finally confirmed after a consensus is reached by two radiation oncologists and a medical physicist. The fractions and radiation doses of Cyberknife depend on each patient's medical condition, as well as the spatial location of the tumour and the adjacent organs at risk. Participants are required to receive SBRT with Cyberknife and one cycle of S-1 simultaneously. After the concurrent radiochemotherapy, two or three cycles of S-1 will be sequentially given. Optimisation of the combination of Cyberknife and S-1 focuses on the interval between Cyberknife and the initial of S-1. However, due to severe adverse effects or those regarded as grade 3 or 4 toxicities, the doses of radiation or S-1 may be modified or the interval of each radiation and the initial of S-1 may be delayed, or the treatment may even be stopped temporarily. These patients would be treated immediately and properly or, if deemed necessary, under the consultation of the multiple disciplinary team. For some patients, if they are reluctant to participate in the trial or are inappropriate for the treatment, as requested or evaluated by investigators, they would withdraw from the study and receive other alternative treatments. Figure 1 illustrates the flow diagram of the study.

\section{Doses of S-1}

The doses of S-1 are calculated by the body surface area. Hence, patients allocated to Cyberknife combined with S-1 arm will receive S-1 orally, two times a day, at a dose of $80 \mathrm{mg} / \mathrm{m}^{2}$ for 28 days, followed by a 14-day interval.

\section{CT simulation for treatment planning}

Each patient should be on fasting for at least 8 hours before the simulation. Vacuum bags are customised with patients in the supine position, according to the patient's body shape for immobilisation during Cyberknife. SBRT is delivered via Cyberknife, an image-guided frameless stereotactic robotic radiosurgery system (Accuray Corporation, Sunnyvale, California, USA), that consists of a linear accelerator mounted on a robot arm with six degrees of freedom. In this system, the 


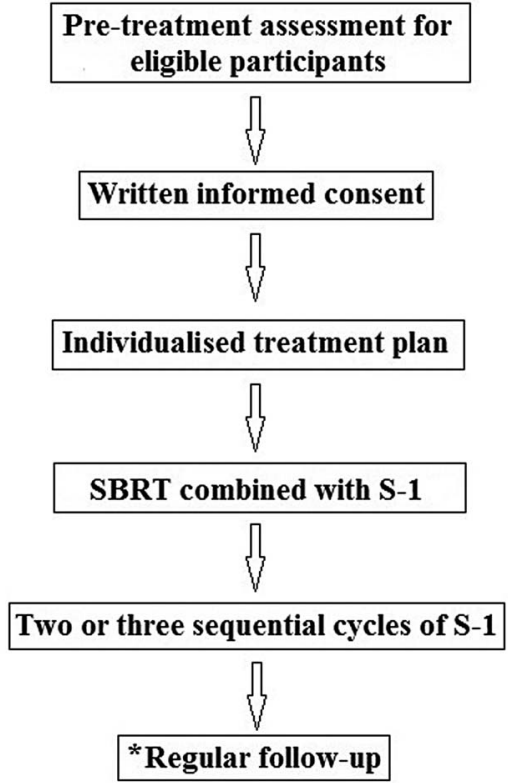

*Whether patients should receive thereafter treatment depends on their follow-up.

Figure 1 Illustrates the flow diagram of the study. SBRT, stereotactic body radiation therapy.

confluence of a large number of non-isocentric pencil beams permits the treatment of irregularly shaped target volumes with rapid dose falloffs. The Cyberknife tracking system automatically compensates for the alignment offset and patient movements by adjusting the treatment isocentre. In addition, a CT-based treatment planning system is used at our institution. Then, plain CT and an enhanced pancreatic parenchymal CT are performed for radiation treatment planning and target delineations. CT images are acquired under breath-hold condition (preferably end-expiratory). Pretreatment diagnostic imaging would be co-registered to the simulation CT in cases where the patient is unable to tolerate intravenous contrast. The scan range includes the whole pancreas, at least $10 \mathrm{~cm}$ above and below the tumour. Spiral CT is performed with a slice thickness of $1.5 \mathrm{~mm}$, and images are reconstructed in slices of $1.5 \mathrm{~mm}$ at most. Intravenous contrast enhancement is performed with the injection of $80-100 \mathrm{~mL}$ of iodixanol, at a flow rate of $2.5 \mathrm{~mL} / \mathrm{s}$, and a delay of $45-55 \mathrm{~s}$; as required for the pancreatic parenchymal phase.

\section{Registration and tracking}

The co-registrations of biphasic CT images are based on fiducials and anatomical (spinal) fusion. Before CT simulation, fiducials should be implanted using endoscopic ultrasound or CT guidance. This is essential for treatment planning and delivery. CT simulation will be performed 7-10 days after fiducial placement. This time interval is required to avoid early fiducial marker displacement or migration. In order to improve the accuracy of the treatment planning, the recommended number of implanted fiducials is preferably close to $3-5$, but not in the tumour. As a result, given that fiducials could simulate the spatial location and displacement of the tumour, which is attributable to respiration, motion tracking should be performed by means of the correlation with these seeds; and fiducial markers render the Synchrony system equipped in Cyberknife feasible. This allows for respiratory motion tracking during irradiation. Nevertheless, patients with high risk of bleeding, abdominal infection, pancreatitis or pancreatic fistula are contradictory to several fiducial implants. Hence, one fiducial plus X-sight spine and Synchrony tracking technique could be alternatively used. Before treatment, direct digital radiography images of the spine would be applied to detect six-dimensional (6-D) errors; and this would be subsequently corrected for X-sight spine tracking on the patient's positioning. This would enable fiducial tracking during treatment.

\section{Treatment planning and target delineation}

After CT simulation, CT images are transferred to the workstation where the target volumes are contoured by an attending radiation oncologist. Gross tumour volume (GTV) is delineated as a radiographically evident gross disease in contrast CT acquired from the portal venous phase. At the discretion of the physician, clinical target volume (CTV) encompassing areas of the potential subclinical disease spread is also designated. In most cases, the CTV equals GTV. A 2-5 mm expansion margin is included to determine the planning target volume (PTV). When the tumour is adjacent to critical organs, the expansion of CTV should be avoided. Therefore, an individualised treatment plan would be developed based on tumour geometries and locations. Ninety per cent of PTV should be covered by the prescription dose. The prescription isodose line is limited to $70-75 \%$, which would restrict the tumour $\mathrm{D}_{\max }$. If the dose level violates the constraint of SBRT, the patient would be considered as ineligible for this trial. A single dose of PTV varies from 6.5 to $9 \mathrm{~Gy}$. In particular, these doses would be reduced if the tumour is approximately one-third or more of the duodenum or stomach circumference, or if the tumour abuts the bowel in only one area, as determined by the relationship of the tumour to the duodenum in axial, coronal and sagittal planes in CT scans, or the space between the tumour and the bowel wall is $<3 \mathrm{~mm}$. Normal tissue constraints are according to the American Association of Physicists in Medicine guidelines in Task Group-101, ${ }^{31}$ as presented in table 2.

\section{Long-term follow-up}

Patients are re-evaluated after Cyberknife every 1 month for the first 3 months, every 2-3 months for the next 2 years and every 6 months for a total 5 years. Remissions of symptoms and radiation-related toxicities would be assessed. In addition, they would undergo laboratory tests, physical examinations, analysis of quality of life, KPS scores and imaging studies every month 
Table 2 Critical structures and threshold doses

\begin{tabular}{|c|c|c|c|}
\hline Organs & \multicolumn{2}{|c|}{ Threshold doses (5 fractions) } & \multirow[t]{2}{*}{ Minimum critical volume below threshold } \\
\hline Parallel organs & & & \\
\hline Liver & $21 \mathrm{~Gy}$ & & $700 \mathrm{cc}$ \\
\hline Kidney & $17.5 \mathrm{~Gy}$ & & $200 \mathrm{cc}$ \\
\hline Organs & Threshold doses (5 fractions) & Maximum point dose & Maximum critical volume above threshold \\
\hline \multicolumn{4}{|l|}{ Serial organs } \\
\hline Spinal cord & $23 \mathrm{~Gy}$ & $30 \mathrm{~Gy}$ & $0.35 \mathrm{cc}$ \\
\hline Duodenum & 18 Gy & 32 Gy & $5 \mathrm{cc}$ \\
\hline Bowel & 19.5 Gy & 35 Gy & $5 \mathrm{cc}$ \\
\hline Stomach & 18 Gy & 32 Gy & $10 \mathrm{cc}$ \\
\hline Oesophagus & $19.5 \mathrm{~Gy}$ & 35 Gy & $5 \mathrm{cc}$ \\
\hline Colon & 25 Gy & 38 Gy & $20 \mathrm{cc}$ \\
\hline
\end{tabular}

within the first 3 months. Subsequent examinations in later follow-ups are the same.

\section{Outcomes}

Outcome definitions

Primary outcomes of the study were OS and 1-year, 2-year, 3-year, 4-year and 5-year OS rates. OS is defined as the time from the date of enrolment to death from any cause. Patients lost to follow-up, withdrawn or alive at the end of the follow-up should be kept confidential.

Secondary outcomes include cancer-specific survival (CSS), PFS, time to progression (TTP), LCR, clinical benefit rates (CBR), radiation-induced acute and late toxicities, adverse effects of chemotherapy and quality of life of the patient. CSS is the time from inclusion to death caused by the tumour. PFS is the time from the date of enrolment to the confirmation of disease progression at any sites or death from any causes, if this occurred before disease progression. Local control is considered as a lack of enlargement of the tumour volume radiographically or stable/declining standardised uptake values on positron emission tomography CT scans. The definition of TTP is the time from inclusion to the recurrence of the tumour, including local recurrence or metastasis. CBR is the ratio of the number of patients with complete response, partial response, or stable disease to the total number of enrolled patients.

\section{Evaluation of outcomes}

PFS, TTP, LCR and CBR are all associated with treatment response, as determined by the Response Evaluation Criteria In Solid Tumors (RECIST) criteria (V.1.1). Quality of life would be measured through EORTC QLQ (QLQ-C30 and QLQ-PAN26). Radiation-induced acute toxicities are adverse effects that occur within 90 days after treatment, and determined by the Radiation Therapy Oncology Group, 'Acute radiation morbidity scoring criteria'. Late toxicities occurring 3 months after
SBRT are evaluated by the Radiation Therapy Oncology Group/EORTC, 'Late radiation morbidity scoring schema'.

\section{Sample size determination}

It was assumed that 1-year OS rate was $70 \%$ of locally advanced pancreatic cancers treated with Cyberknife combined with S-1. The potential benefit of the regimen was $\sim 20 \%$ increase in 1-year OS rate. In order to have $90 \%$ power to reject the null hypothesis if the alternative was true at level $\alpha=0.05$, the required sample size for our study was 138 . Additionally, a loss of $20 \%$ of patients due to lost to follow-up or withdrawn due to adverse effects or other reasons was estimated. Hence, a total of 190 patients were enrolled into this study.

\section{Data analysis}

Statistical testing will be performed using SPSS V.20.0 (IBM Corp, Armonk, New york, USA). All outcomes would be analysed based on intention-to-treat principle. PFS, OS and local control are calculated via the Kaplan-Meier method compared by the log-rank (Mantel-Cox) test. Response rates would be compared by a test of proportions. Further analysis would be performed in subgroups stratified by different factors.

\section{Data management and quality assurance}

Data regarding the patient's characteristics, medical histories and results of clinical and laboratory tests or examinations will be kept in a password-protected database at the Department of Radiation Oncology in Changhai Hospital, which will only be disclosed to authorised individuals. The Ethics Committee of Changhai Hospital will be responsible for data monitoring. In addition, trial conducts will be audited by the committee every 6 months after the recruitment of participants. The accuracy of the data entry into the database will be confirmed by two administrators. The interim results will be accessed to authorised individuals and reported to the Ethics Committee of Changhai Hospital, 
which would make the final decision to terminate the trial if severe adverse effects occur frequently.

\section{DISCUSSION}

S-1 has been considered as an important chemotherapeutic drug in pancreatic cancer. In addition to convenient oral medication, many studies have verified that S-1 was not inferior to gemcitabine regarding OS and PFS. In adjuvant therapy, S-1 may be a candidate drug for a patient refractory to gemcitabine, but without phase III clinical trials. In addition, radiotherapy combined with S-1 probably contributed to the down staging of the tumour, as well as the lower complication rates of surgeries as the neoadjuvant therapy. ${ }^{32}{ }^{33}$ It was elucidated that neoadjuvant radiotherapy with S-1 was beneficial to potential candidates for radical surgeries, because OS could be improved significantly. ${ }^{33}$

SBRT with Cyberknife has been proven with lower radiation toxicities, higher accuracy and better efficacy compared with conventional radiotherapy. Therefore, it is pivotal to evaluate the efficacy of SBRT with S-1 as the initial or even neoadjuvant treatment in locally advanced pancreatic cancer, which may be not inferior to previous conventional treatment or even more beneficial to patients than standard chemotherapy with conventional radiotherapy. However, no prospective clinical trials have provided such investigation. Hence, the goal of the SILAPANC trial is to assess whether better prognosis could be achieved with Cyberknife combined with S-1 followed by sequential S-1 as an initial treatment, which may provide new insights into the treatment of locally advanced pancreatic cancer.

\section{Author affiliations}

Changhai Hospital Affiliated to Second Military Medical University, Shanghai, China

Contributors $\mathrm{HZ}$ was the primary investigator of the study. $\mathrm{XZ}$ and $\mathrm{XJ}$ designed the protocol and $\mathrm{HZ}$ amended it. $\mathrm{XZ}, \mathrm{FC}$ and $\mathrm{FF}$, were responsible for recruitment of participants. $S Q$ and $Y S$ were responsible for data entry. ZJ and $Y C$ were responsible for data analysis.

Competing interests None declared.

Patient consent Obtained.

Ethics approval Changhai Hospital Ethics Committee.

Provenance and peer review Not commissioned; externally peer reviewed.

Data sharing statement The unpublished data from the study are available in peer-review journals and conferences.

Open Access This is an Open Access article distributed in accordance with the Creative Commons Attribution Non Commercial (CC BY-NC 4.0) license, which permits others to distribute, remix, adapt, build upon this work noncommercially, and license their derivative works on different terms, provided the original work is properly cited and the use is non-commercial. See: http:// creativecommons.org/licenses/by-nc/4.0/

\section{REFERENCES}

1. Siegel R, Naishadham D, Jemal A. Cancer statistics, 2012. CA Cancer J Clin 2012;62:10-29.

2. Sener SF, Fremgen A, Menck HR, et al. Pancreatic cancer: a report of treatment and survival trends for 100313 patients diagnosed from
1985-1995 using the national cancer database. J Am Coll Surg 1999;189:1-7.

3. Richter A, Niedergethmann M, Sturm JW, et al. Long-term results of partial pancreaticoduodenectomy for ductal adenocarcinoma of the pancreatic head: 25-year experience. World J Surg 2003;27:324-9.

4. Tseng JF, Raut CP, Lee JE, et al. Pancreaticoduodenectomy with vascular resection: margin status and survival duration. J Gastrointest Surg 2004;8:935-49.

5. Hoyer M, Roed H, Sengelov L, et al. Phase-Il study on stereotactic radiotherapy of locally advanced pancreatic carcinoma. Radiother Oncol 2005;76:48-53.

6. Burris HA III, Moore MJ, Andersen J, et al. Improvements in survival and clinical benefit with gemcitabine as first-line therapy for patients with advanced pancreas cancer: a randomized trial. J Clin Oncol 1997;15:2403-13.

7. Ueno H, Okusaka T, Ikeda M, et al. An early phase II study of S-1 in patients with metastatic pancreatic cancer. Oncology 2005;68:171-8.

8. Ueno $\mathrm{H}$, loka $\mathrm{T}$, Ikeda $\mathrm{M}$, et al. Randomized phase III study of gemcitabine plus S-1, S-1 alone, or gemcitabine alone in patients with locally advanced and metastatic pancreatic cancer in Japan and Taiwan: GEST study. J Clin Oncol 2013;31:1640-8.

9. Morizane C, Okusaka T, Furuse J, et al. A phase II study of S-1 in gemcitabine-refractory metastatic pancreatic cancer. Cancer Chemother Pharmacol 2009;63:313-19.

10. Sudo K, Yamaguchi T, Nakamura K, et al. Phase II study of S-1 in patients with gemcitabine-resistant advanced pancreatic cancer. Cancer Chemother Pharmacol 2011;67:249-54.

11. Mizuno N, Yamao K, Komatsu Y, et al. Randomized phase II trial of S-1 versus S-1 plus irinotecan (IRIS) in patients with gemcitabine refractory pancreatic cancer. J Clin Oncol 2012;30:abstr 263.

12. Ohkawa $\mathrm{S}$, Okusaka $\mathrm{T}$, Isayama $\mathrm{H}$, et al. Randomised phase II trial of S-1 plus oxaliplatin vs S-1 in patients with gemcitabine-refractory pancreatic cancer. Br J Cancer 2015;112:1428-34.

13. Ge F, Xu N, Bai Y, et al. S-1 as monotherapy or in combination with leucovorin as second-line treatment in gemcitabine-refractory advanced pancreatic cancer: a randomized, open-label, multicenter, phase II study. Oncologist 2014;19:1133-4.

14. Rich TA, Shepard RC, Mosley ST. Four decades of continuing innovation with fluorouracil: current and future approaches to fluorouracil chemoradiation therapy. J Clin Oncol 2004;22:2214-32.

15. Moningi S, Marciscano AE, Rosati LM, et al. Stereotactic body radiation therapy in pancreatic cancer: the new frontier. Expert Rev Anticancer Ther 2014;14:1461-75.

16. Berber B, Sanabria JR, Braun K, et al. Emerging role of stereotactic body radiotherapy in the treatment of pancreatic cancer. Expert Rev Anticancer Ther 2013;13:481-7.

17. Chuong MD, Springett GM, Freilich JM, et al. Stereotactic body radiation therapy for locally advanced and borderline resectable pancreatic cancer is effective and well tolerated. Int $J$ Radiat Oncol Biol Phys 2013;86:516-22.

18. Herman JM, Chang DT, Goodman KA, et al. Phase 2 multi-institutional trial evaluating gemcitabine and stereotactic body radiotherapy for patients with locally advanced unresectable pancreatic adenocarcinoma. Cancer 2015;121:1128-37.

19. Mahadevan A, Jain S, Goldstein M, et al. Stereotactic body radiotherapy and gemcitabine for locally advanced pancreatic cancer. Int J Radiat Oncol Biol Phys 2010;78:735-42.

20. Koong AC, Le QT, Ho A, et al. Phase I study of stereotactic radiosurgery in patients with locally advanced pancreatic cancer. Int J Radiat Oncol Biol Phys 2004;58:1017-21.

21. Koong AC, Christofferson E, Le QT, et al. Phase II study to assess the efficacy of conventionally fractionated radiotherapy followed by a stereotactic radiosurgery boost in patients with locally advanced pancreatic cancer. Int J Radiat Oncol Biol Phys 2005;63:320-3.

22. Schellenberg D, Goodman KA, Lee F, et al. Gemcitabine chemotherapy and single-fraction stereotactic body radiotherapy for locally advanced pancreatic cancer. Int J Radiat Oncol Biol Phys 2008;72:678-86.

23. Schellenberg D, Kim J, Christman-Skieller C, et al. Single-fraction stereotactic body radiation therapy and sequential gemcitabine for the treatment of locally advanced pancreatic cancer. Int $J$ Radiat Oncol Biol Phys 2011:81:181-8.

24. Mahadevan A, Miksad R, Goldstein M, et al. Induction gemcitabine and stereotactic body radiotherapy for locally advanced nonmetastatic pancreas cancer. Int J Radiat Oncol Biol Phys 2011;81:e615-22. 
25. Polistina F, Costantin G, Casamassima F, et al. Unresectable locally advanced pancreatic cancer: a multimodal treatment using neoadjuvant chemoradiotherapy (gemcitabine plus stereotactic radiosurgery) and subsequent surgical exploration. Ann Surg Oncol 2010;17:2092-101.

26. Rwigema JC, Parikh SD, Heron DE, et al. Stereotactic body radiotherapy in the treatment of advanced adenocarcinoma of the pancreas. Am J Clin Oncol 2011;34:63-9.

27. Gurka MK, Collins SP, Slack R, et al. Stereotactic body radiation therapy with concurrent full-dose gemcitabine for locally advanced pancreatic cancer: a pilot trial demonstrating safety. Radiat Oncol 2013;8:44.

28. Didolkar MS, Coleman CW, Brenner MJ, et al. Image-guided stereotactic radiosurgery for locally advanced pancreatic adenocarcinoma results of first 85 patients. J Gastrointest Surg 2010;14:1547-59.
29. Goyal K, Einstein D, Ibarra RA, et al. Stereotactic body radiation therapy for nonresectable tumors of the pancreas. J Surg Res 2012;174:319-25.

30. NCCN guidelines 'Pancreatic adenocarcinoma', version 2.2015.

31. Benedict SH, Yenice KM, Followill D, et al. Stereotactic body radiation therapy: the report of AAPM Task Group 101. Med Phys 2010;37:4078-101.

32. Breslin TM, Hess KR, Harbison DB, et al. Neoadjuvant chemoradiotherapy for adenocarcinoma of the pancreas: treatment variables and survival duration. Ann Surg Oncol 2001;8:123-32.

33. Laurence JM, Tran PD, Morarji K, et al. A systematic review and meta-analysis of survival and surgical outcomes following neoadjuvant chemoradiotherapy for pancreatic cancer. J Gastrointest Surg 2011;15:2059-69. 